\title{
Improvement of the Covergence of the Learning Using the Modified Back-propagation Method
}

\author{
Masato SEKIt and Shinsaku MORItt
}

\author{
† TOYOTA MOTOR CORPORATION \\ 1 Toyota-cho, Toyota, Aichi, 471 JAPAN \\ Phone: +81565 282121 \\ †† Dept.of Electrical Engineeriing, Keio University \\ 3-14-1 Hiyoshi, Kouho, Yokohama, 223 JAPAN \\ Phone: +81 455631141 Ext. 3319 FAX: +81 455632773 \\ email: mori@mori.elec.keio.ac.jp
}

\begin{abstract}
The modified Back-Propagation(BP) method for improving the convergence of the learning is proposed. Our modification consists of two changes. 1) For accelerating the learning near convergence, we use a new type of activation function instead of a sigmoid function. 2) In order to accelerate the learning speed more effectively, we consider the role of momentum term and a propose a method of setting up the momentum rate. Though the momentum term is the heuristic method for the acceleration of the convergence and is customary used, there are few discussions for the momentum term. Using the proposed method, we carry out the computer simulations for two example probrems. We confirm that both of the convergence speed and convergence rate are improved.
\end{abstract}

\section{Actuvation function}

Generally, a continuous and nonlinear activation function is needed for the BP, and the sigmoid function has been used. But when the condition of network is near convergence, the characteristic of sigmoid function leads the small error signal and the slow learning speed. To recover the defect of the characteristic of the sigmoid function, we used the following activation function.

$$
y=f(x)=\left\{\begin{array}{cc}
0 & (x \leq-a) \\
k(x+a)^{2} & (-a<x \leq 0) \\
-k(x-a)^{2}+1 & (0<x \leq a) \\
1 & (x>a)\left(2 k a^{2}=1\right)
\end{array}\right.
$$

\section{Momentum term}

For setting up the momentum rate, the basic concept of the BP that is the start point of the learning is output layer is one of the important factors. Here we proposed the method that the momentum rate at the output layer is set up larger than that of hidden layer. That is we try to urge accelerating at the output layer. This will connect to accelerate at the hidden layer. When the error surface seems to be long slope without $\mathrm{V}$-shaped varrey, the larger step size for weight change is the more effective for accelerating. On the other and the learning in the oscillation, the momentum term operates to reduce it. Then we propose the following method.

Case1: When the signs of the learning and momentum term are the same, we use the larger $\alpha$.

Case2: When the signs of the learning and momentum term are different, we use the larger $\alpha$.

$$
\text { Conlusions }
$$

From the simulation results, we recognized the following facts. The proposed activation function accelerates the learning near convergence. The method of casel for the moentum rate is effective at the middle stage of learning. The method of case 2 is also effective at the early stage. As such we succeedin reducing the number of the learning steps at all of the stages. In addition, the convergence rate of the network is also getting better.

\section{References}

[1] D.E.Rumelhart, et al.:Parallel Distributed Processing, voll, pp.318-362, MA: MIT Press, 1986.

[2] T.P.Vogl, et al.: "Accelerating the convergence of the Back-Propagation Method", Biol. Cybern. 59, pp. 257-263, 1988.

[3] T.Yoshikawa, Y.Kawaguchi: "A High Speed Method for the Back-Propagation Rules in Neural Network", Trans. of the IEICE, vol. J75-DII, no.4, pp. 837-840, April, 1992. 